\title{
Sistema web de solicitudes de acceso a la información
}

\section{Web system of requests for access to information}

\author{
RAFAEL-PÉREZ, Eva†๋*, HERNÁNDEZ-BOLAÑOS, Alan Jhaseel, LÓPEZ-CRUZ, Eliel y \\ MORALES-HERNÁNDEZ, Maricela
}

\begin{abstract}
Instituto Tecnológico de Oaxaca, Departamento de Sistemas y Computación. Avenida Ing. Víctor Bravo Ahuja No. 125 Esquina Calzada Tecnológico, C.P. 68030, México.
\end{abstract}

ID $1^{\text {er }}$ Autor: Eva, Rafael-Pérez / ORC ID: 0000-0003-2793-1254, CVU CONACYT ID: 905268

ID $1^{\text {er }}$ Coautor: Alan Jhaseel, Hernández-Bolaños / ORC ID: 0000-0002-3997-2400, CVU CONACYT ID: 1086535

ID $2^{\text {do }}$ Coautor: Eliel López Cruz / ORC ID: 0000-0002-8282-0410, CVU CONACYT ID: 1086533

ID $3^{\text {er }}$ Coautor: Maricela, Morales-Hernández / ORC ID: 0000-0002-3521-2041, CVU CONACYT ID: 731036

DOI: $10.35429 / \mathrm{JOCT} .2020 .13 .4 .14 .23$

Recibido: Abril 20, 2020; Aceptado: Junio 30, 2020

\begin{abstract}
Resumen
El desarrollo del proyecto Sistema Web de Solicitudes de Acceso a la Información para la Secretaría de la Contraloría y Transparencia Gubernamental del Estado de Oaxaca, tiene como objetivo contribuir al control interno de las Solicitudes de Acceso a la Información que son enviadas a las Dependencias, Entidades, Órganos Auxiliares y Fideicomisos que integran la Administración Pública Estatal, para que se de atención a las Solicitudes y se cumplan dentro de los plazos establecidos por la Ley de Transparencia y Acceso a la Información Pública para el Estado de Oaxaca (LGTAIPO). El sistema cuenta con los módulos de Solicitudes, Dependencias, historial de solicitudes y respuestas, reportes, tiene un chat interno y permite agendar las reuniones. Con este proyecto se contribuye a eficientar y automatizar los procesos, e incrementar la capacidad de respuesta en la atención a las solicitudes. El sistema web se basó en la metodología de desarrollo ágil en Programación Extrema (XP), se utilizó el gestor de base de datos en MySql y el lenguaje de programación GoLand para su desarrollo.
\end{abstract}

\begin{abstract}
The Secretariat of the Comptroller and Government Transparency of the State of Oaxaca (SCTGEO) has an objective with the development of the project called Web System of Requests for Access to Information. This objective is to contribute to internal control of the Requests for Access to Information that are sent to the dependencies, entities, auxiliary bodies, and, escrows that integrate State Public Administration. It also follows up that the Requests are attended to and are fulfilled within the terms established by the Law of Transparency and Access to Public Information for the State of Oaxaca (LGTAIPO). The web system has the following functionalities: a history of requests and responses, it allows the use of different response formats; it generates reports based on a period defined by the user; it allows you to schedule meetings, and it follows up if necessary. In addition, it has a Request, Dependencies module and an internal chat so that they can communicate with each other. The web system was based on the agile development model using Extreme Programming (XP) and the MySql database manager was used.
\end{abstract}

Information System, Requests, Dependencies

Citación: RAFAEL-PÉREZ, Eva, HERNÁNDEZ-BOLAÑOS, Alan Jhaseel, LÓPEZ-CRUZ, Eliel y MORALESHERNÁNDEZ, Maricela. Sistema web de solicitudes de acceso a la información. Revista de Tecnologías Computacionales. 2020. 4-13:14-23.

\footnotetext{
* Correspondencia del Autor (Correo Electrónico: evarafaelperez@ gmail.com)

$\dagger$ Investigador contribuido como primer autor.
} 


\section{Introducción}

Desde el surgimiento de la computación, los sistemas de información (SI) y las tecnologías asociadas han cambiado en forma significativa la forma como las empresas y las personas organizan, dirigen, controlan y planean sus negocios (Avison y Pries-Heje, 2005), un sistemas de información se refiere al conjunto de personas, datos, procesos y tecnología de la información que interactúan para recoger, procesar, almacenar y proveer la información necesaria para el correcto funcionamiento de la organización, donde intervienen las personas, los datos, procesos y las tecnologías de información (Whitten, Bentley y Dittman, 2004); por otro lado, las Tecnologías de la Información y Comunicación (TIC), hoy en día juegan un papel determinante en el uso e implantación de los sistemas, de tal manera que permiten eficientar los procesos, compartir los recursos, aumentar la productividad y la toma de decisiones.

El proyecto denominado Sistema Web de Solicitudes de Acceso a la Información, fue desarrollado para la Secretaría de Contraloría y Transparencia Gubernamental con el objetivo de eficientar el control interno de las solicitudes que se presentan en cada una de las Dependencias, Entidades, Órganos Auxiliares y Fideicomisos que integran la Administración Pública Estatal, y contribuye en gran medida a la gestión y el seguimiento en el trámite de las solicitudes, es importante mencionar que el sistema web únicamente permite automatizar los procesos de las solicitudes. En relación a la publicación de la información solicitada, es otro proceso diferente que la Secretaría de la Contraloría lo determina según corresponda.

El sistema web fue desarrollado bajo la metodología en programación extrema, utilizando el lenguaje de programación Dart y cuenta con los siguientes módulos: Usuarios, Dependencias, Solicitudes, Seguimiento, Clasificación, Historial de solicitudes y respuestas, Reportes, Notificaciones, Reuniones $\mathrm{y}$ un Chat interno.

Por lo anterior el presente trabajo abarca los siguientes puntos: Planteamiento del problema, Metodología de desarrollo, Desarrollo del Proyecto, Conclusiones y Referencias.

\section{Planteamiento del problema}

Debido a la gran cantidad de solicitudes que se reciben en las diferentes dependencias, se presentan diversos problemas; por ejemplo, descontrol de las solicitudes que se reciben, hace falta un seguimiento adecuado debido a que cuando se solicita la información por parte del ciudadano, puede solicitar la misma información a dos o más dependencias, lo que provoca que al momento de dar respuesta, estas sean diferentes debido a la falta de homologación de criterios y a que cada dependencia maneja su propia información o que la información no este actualizada; no existe un formato estandarizado, la clasificación de solicitudes se lleva de forma manual, lo que provoca mayor esfuerzo y retrasos para dar respuesta a las diferentes solicitudes como corresponde; los reportes anuales que se generan se realizan de forma manual, lo que provoca inconsistencias en los datos, retrasos, reportes incompletos o que en su defecto no sean entregados en tiempo y forma.

\section{Metodología de desarrollo}

Para la creación de software existen diferentes metodologías de desarrollo a utilizar, Maddison (1983) define a la metodología de software como el conjunto de filosofías, fases, procedimientos, reglas, técnicas, herramientas, documentación y aspectos de formación para los desarrolladores de sistemas de información. Es importante mencionar que no todas las metodologías se adaptan a todos los proyectos, ya que va a depender de las necesidades particulares de cada caso, para el proyecto de software Sistema Web de Solicitudes de Acceso a la Información se utilizó la metodología de desarrollo ágil en Programación Extrema (XP Extreme Programming); propuesto por Kent Beck en 1999, tiene como objetivo la satisfacción del cliente y optimiza el tiempo de desarrollo; la programación extrema se apoya en valores, como la comunicación, la retroalimentación, respeto, simplicidad y coraje, los cuales enfatizan la esencia colaborativa del equipo; cuenta con las fases de análisis, diseño, codificación y pruebas. 


\section{Desarrollo del proyecto}

El desarrollo del sistema web se basó en dos iteraciones que se realizaron según las fases de la metodología en programación extrema. En la fase de Análisis se realizó un plan de reuniones para definir las tareas, los avances del sistema y las fechas de revisión, para esta actividad fue esencial la interacción y la comunicación con el asesor externo, en la tabla 1 se aprecia con más detalle.

\begin{tabular}{|r|l|r|}
\hline \multicolumn{1}{|c|}{ No. Actividad } & Fecha \\
\hline 1 & $\begin{array}{l}\text { Definir la lista maestra de } \\
\text { iteraciones }\end{array}$ & 051019 \\
\hline 2 & $\begin{array}{l}\text { Entrega del avance módulo de } \\
\text { usuarios }\end{array}$ & 101019 \\
\hline 3 & $\begin{array}{l}\text { Entrega del avance módulo de } \\
\text { Dependencias }\end{array}$ & 171019 \\
\hline 4 & $\begin{array}{l}\text { Entrega del avance módulo de } \\
\text { Catalogo de usuarios }\end{array}$ & 241019 \\
\hline 5 & $\begin{array}{l}\text { Avance módulo de Catalogo de } \\
\text { Dependencias }\end{array}$ & 311019 \\
\hline 6 & $\begin{array}{l}\text { Revisar el funcionamiento del } \\
\text { Chat interno. }\end{array}$ & 070117 \\
\hline 7 & Entrega: Primera iteración & 141119 \\
\hline 8 & $\begin{array}{l}\text { Revisar el funcionamiento del } \\
\text { módulo de Solicitudes }\end{array}$ & 211119 \\
\hline 9 & Seguimiento de solicitud. & 131219 \\
\hline 10 & Historial de respuesta & 100120 \\
\hline 11 & Notificaciones, & 230120 \\
\hline 12 & Módulo de reuniones & 300120 \\
\hline 13 & Generación de reportes. \\
\hline 14 & Entrega: Segunda iteración & 040220 \\
\hline 15 & Entrega del proyecto final \\
\hline
\end{tabular}

Tabla 1 Plan de reuniones

Fuente: Elaboración propia

En la tabla 2 se muestra la lista maestra de iteraciones y las tareas que fueron definidas para el desarrollo del proyecto.

\begin{tabular}{|l|l|}
\hline \multicolumn{2}{|c|}{ Lista Maestra de Iteraciones } \\
\hline No. & \multicolumn{1}{|c|}{\begin{tabular}{c}
\multicolumn{1}{|c|}{ Primera Iteración } \\
Módulos
\end{tabular}} \\
\hline 1. & $\begin{array}{l}\text { Inicio de sesión: Diseñar el módulo para el } \\
\text { ingreso de los usuarios. }\end{array}$ \\
\hline 2. & $\begin{array}{l}\text { Usuarios: Realizar el diseño y codificación para } \\
\text { agregar, eliminar, editar y visualizar }\end{array}$ \\
\hline 3. & $\begin{array}{l}\text { Dependencias. Realizar el diseño y codificación } \\
\text { para agregar, eliminar, editar y visualizar las } \\
\text { dependencias. }\end{array}$ \\
\hline 4. & $\begin{array}{l}\text { Catálogo de usuario. Diseñar y codificar la } \\
\text { interfaz para mostrar el catálogo de los usuarios } \\
\text { existentes }\end{array}$ \\
\hline 5. & $\begin{array}{l}\text { Catálogo de dependencia. Diseñar y codificar la } \\
\text { interfaz para mostrar el catálogo de las } \\
\text { dependencias. }\end{array}$ \\
\hline 6. & $\begin{array}{l}\text { Chat interno. Desarrollar el chat para enviar, } \\
\text { recibir y listar los mensajes enviados entre las } \\
\text { dependencias. }\end{array}$ \\
\hline
\end{tabular}

\begin{tabular}{|l|l|}
\hline \multicolumn{3}{|c|}{ Segunda Iteración } \\
\hline 7. & $\begin{array}{l}\text { Módulo de solicitudes. Diseñar y codificar la } \\
\text { interfaz para agregar, editar y mostrar las } \\
\text { solicitudes }\end{array}$ \\
\hline 9. & $\begin{array}{l}\text { Seguimiento de solicitud. Diseñar el módulo para } \\
\text { visualizar el historial de los movimientos de las } \\
\text { solicitudes }\end{array}$ \\
\hline 10. & $\begin{array}{l}\text { Historial de respuesta, diseñar y codificar el } \\
\text { módulo para visualizar opciones de respuesta para } \\
\text { una pregunta y seleccionar según corresponda. }\end{array}$ \\
\hline 11. & $\begin{array}{l}\text { Notificaciones, diseñar y codificar una interfaz } \\
\text { que permita enviar las notificaciones } \\
\text { correspondientes }\end{array}$ \\
\hline $\begin{array}{l}\text { Módulo de reuniones. Diseñar y codificar una } \\
\text { interfaz que permita solicitar, crear, editar, } \\
\text { eliminar y listar las reuniones }\end{array}$ \\
\hline 12. & $\begin{array}{l}\text { Generación de reportes. Diseñar y codificar una } \\
\text { interfaz gráfica para generar los reportes de los } \\
\text { informes anuales y un archivo Excel para su } \\
\text { descarga. }\end{array}$ \\
\hline
\end{tabular}

Tabla 2 Lista maestra de iteraciones y tareas Fuente: Elaboración propia

Para la definición de los requerimientos funcionales y no funcionales, se utilizó la técnica de historias de usuario que se aplican en la mayoría de los métodos ágiles, son una descripción breve de una funcionalidad software tal y como la percibe el usuario, (Cohn, 2004); es importante mencionar que desarrollaron diferentes historias de usuario en cada iteración. En la tabla 3, se aprecian los campos que se consideran más necesarios para describir de una manera adecuada la historia de usuario, como son:

- ID: 06, identificador de la historia de usuario, único para la funcionalidad o trabajo.

Título: Registrar usuarios, título descriptivo de la historia de usuario.

- Descripción: Como usuario administrador quiero registrar usuarios para tener la información actualizada, descripción sintetizada de la historia de usuario con base al patrón: Como [rol del usuario], quiero [objetivo], para poder [beneficio], que (Cohn, 2004) recomienda, ya que garantiza que la funcionalidad está descrita a un alto nivel y de manera no demasiado extensa.

- Criterio de validación: pruebas de aceptación consensuadas con el cliente o usuario, en este ejemplo se valida la petición del usuario. 
Historia de Usuario

\begin{tabular}{|l|l|}
\hline \multicolumn{2}{|c|}{ Historia de Usuario } \\
\hline ID: 6 & Usuario: Administrador \\
\hline Título de la historia: Registrar usuarios \\
\hline $\begin{array}{l}\text { Prioridad en } \\
\text { negocio: media }\end{array}$ & Riesgo en desarrollo: Baja \\
\hline Puntos estimados: 2 & Módulo: Usuarios \\
\hline $\begin{array}{l}\text { Descripción: } \\
\text { Como usuario administrador quiero registrar usuarios para } \\
\text { tener la información actualizada }\end{array}$ \\
\hline $\begin{array}{l}\text { Validación } \\
\text { El usuario administrador puede registrar usuarios y } \\
\text { asignarlos a una dependencia, cada usuario puede tener } \\
\text { solo una dependencia, los datos requeridos son registrados } \\
\text { por el usuario al ingresar por primera vez al sistema. }\end{array}$ \\
\hline
\end{tabular}

Tabla 3 Historia de usuario

Fuente: Elaboración propia

Otra actividad que se realizó fue la identificación de los tipos de usuarios con sus roles y privilegios para el acceso al sistema:

El usuario Administrador, tiene el acceso total a cada uno de los módulos del sistema web, es responsable de registrar dependencias y usuarios, asigna dependencias a los usuarios registrados para llevar el control de las solicitudes, asigna las contraseñas a usuarios, autoriza y planea las reuniones.

- Usuario dependencia. Este usuario es responsable de registrar las solicitudes de acceso a la información que sean dirigidas a la dependencia, es el encargado de dar seguimiento al proceso para brindar respuesta a la solicitud de información y solicita las reuniones en caso de que sean necesarias para resolver dudas de las solicitudes.

- Usuario Auditor, solo tiene acceso de consulta de información de las solicitudes que llegan a cada dependencia y saber el estado actual de cada solicitud.

SuperUsuario. Es el usuario que tiene acceso para realizar los respaldos de la base de datos y dar mantenimiento al sistema.
En la fase de diseño, se elaboró el modelo de datos utilizando para ello el diagrama entidad-relación como se aprecia en la figura 1, donde se establece la relación entre las diferentes entidades con sus atributos, así como la relación entre pares de entidades a través de un verbo y que se asocian mediante la cardinalidad $(0,1)$ $(1,1),(1, n)$ o $(n, n)$ a través de un campo clave denominado llave primaria (Silberschatz, Korth y Sudarshan, 2006). Por ejemplo, se establece una relación entre la entidad Solicitud y la entidad Dependencia, cuya cardinalidad es 1 a n, ya que la Dependencia registra $\mathrm{n}$ cantidades de solicitudes; y de la entidad Dependencia a la entidad Solicitud la cardinalidad es de $1 \mathrm{a} 1$, ya que una Solicitud solamente puede estar registrada en una sola Dependencia.

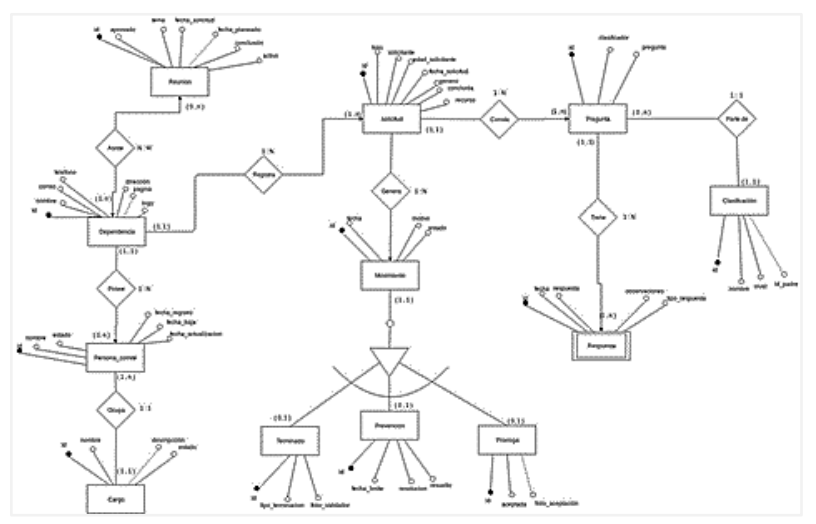

Figura 1 Diagrama Entidad Relación Fuente: Elaboración propia

En cuanto al diseño arquitectónico del sistema web se utilizó el Modelo Vista Controlador (MVC) para clasificar la información, la lógica del sistema y la interfaz que se le presenta al usuario, en la figura número 2 se muestra el funcionamiento del modelo, cuando el usuario realiza una petición al controlador, este recibe dicha petición de la interfaz gráfica y procede a ejecutar la acción pertinente enviando los datos al modelo que contiene la lógica de negocios, el modelo realiza las modificaciones en la base de datos a través del Sistema Gestor de base de datos MySql, luego, devuelve al controlador los cambios solicitados que funciona como intermediario para gestionar el flujo de datos entre el modelo y la vista, cuando el controlador recibe todos los datos del cambio, envía una respuesta a la vista, que contiene las interfaces con las que el usuario tiene interacción, finalmente la vista aplica la modificación en la interfaz del navegador mostrando el resultado al usuario. 


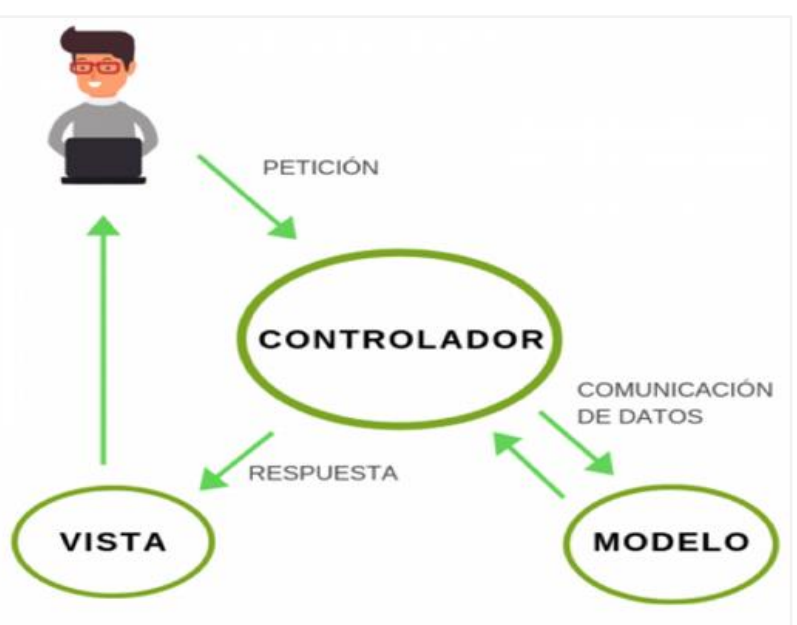

Figura 2 Modelo vista Controlador Fuente: https://nicobobb.com/mvc/

Para el diseño de las interfaces, éstas se fueron generando conforme a la iteración correspondiente, en la figura 3 se muestra la interfaz para agregar una Dependencia, los campos nombre y usuario son datos requeridos, una vez rellenado el formulario, se da clic en la opción Registrar Dependencia, que despliega una pantalla que lista las dependencias registradas.

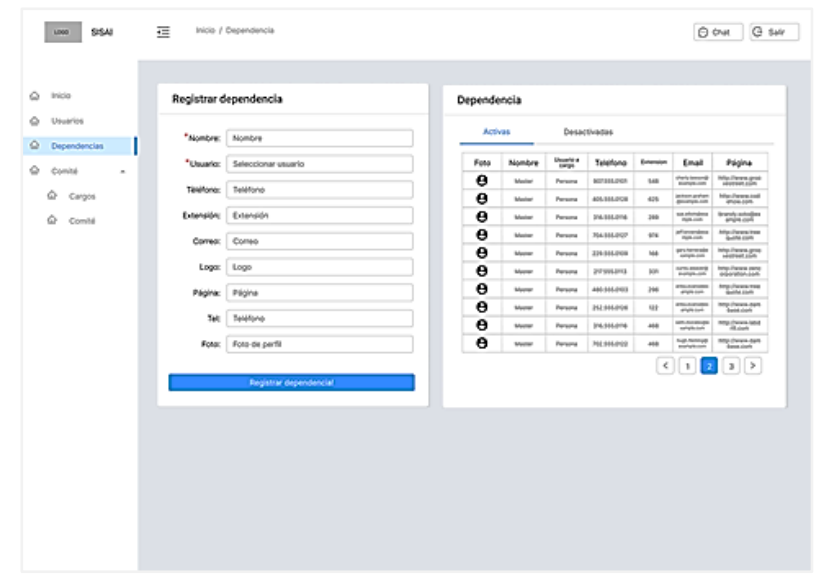

Figura 3 Interfaz gráfica del módulo Dependencia Fuente: Elaboración propia

El sistema web se basó en modelo cliente-servidor; como se observa en la figura 4, por un lado está el cliente (el navegador, el sistema web) quien realiza las peticiones $\mathrm{o}$ solicitudes y por otro lado el servidor (el servidor web); que se encarga de enviar la respuesta a las peticiones solicitadas por el clientes, en donde una única computadora aloja el servicio del Protocolo de transferencia de hipertexto (HTTP), la lógica de negocio que son las especificaciones de la aplicación, la lógica de datos que es la forma como se accede a los datos y los datos propiamente.

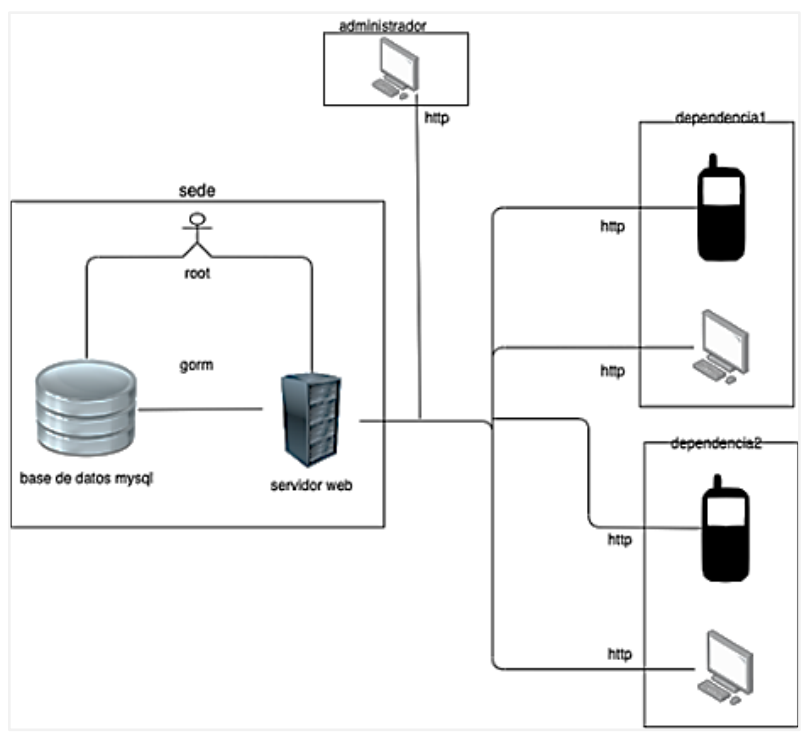

Figura 4 Modelo cliente-servidor Fuente: Elaboración propia

La codificación es un proceso que se realiza en forma paralela con el diseño, la cual está sujeta a las características y fortalezas de la programación extrema, como la rotación de los programadores o la programación en parejas; para la codificación de los módulos del sistema, se utilizó el lenguaje de programación GoLang, es un IDE multiplataforma especialmente integrado para desarrolladores de Go, ya que facilita la lectura, la escritura y la modificación del código Go, un IDE es un Entorno de Desarrollo Integrados, son programas que facilitan la escritura de código para el desarrollo de aplicaciones.

Se codificaron los módulos de: Inicio de sesión, Usuarios, Dependencias, Catálogo de usuarios, Catálogo de dependencias y el Chat interno, Solicitudes, Seguimiento, Historial de respuesta, Notificaciones y Reportes.

En la fase de Pruebas, se aplicaron las pruebas a cada módulo, con las pruebas de integración, se comprobó que los módulos y elementos que interactúan en el sistema funcionaran de manera correcta; con las pruebas de compatibilidad, el sistema web se probó en diferentes navegadores, Mozilla Firefox, Google Chrome, Safari, Opera, y finalmente las pruebas de aceptación que significa la satisfacción del usuario final con el software desarrollado. 


\section{Resultados}

El resultado del desarrollo del Sistema Web de Solicitudes de Acceso a la Información es el diseño y codificación de cada uno de los módulos que se desarrollaron, lo que va a permitir el funcionamiento del sistema como un todo para eficientar y automatizar los procesos de las solicitudes de la Secretaría, como se describen a continuación:

Pantalla de acceso al sistema; se otorgaron los permisos de acceso de acuerdo a los tipos de usuarios, cada usuario cuenta con un nombre de usuario y una contraseña, datos requeridos para el acceso al sistema web, como se ve en la figura 5 .

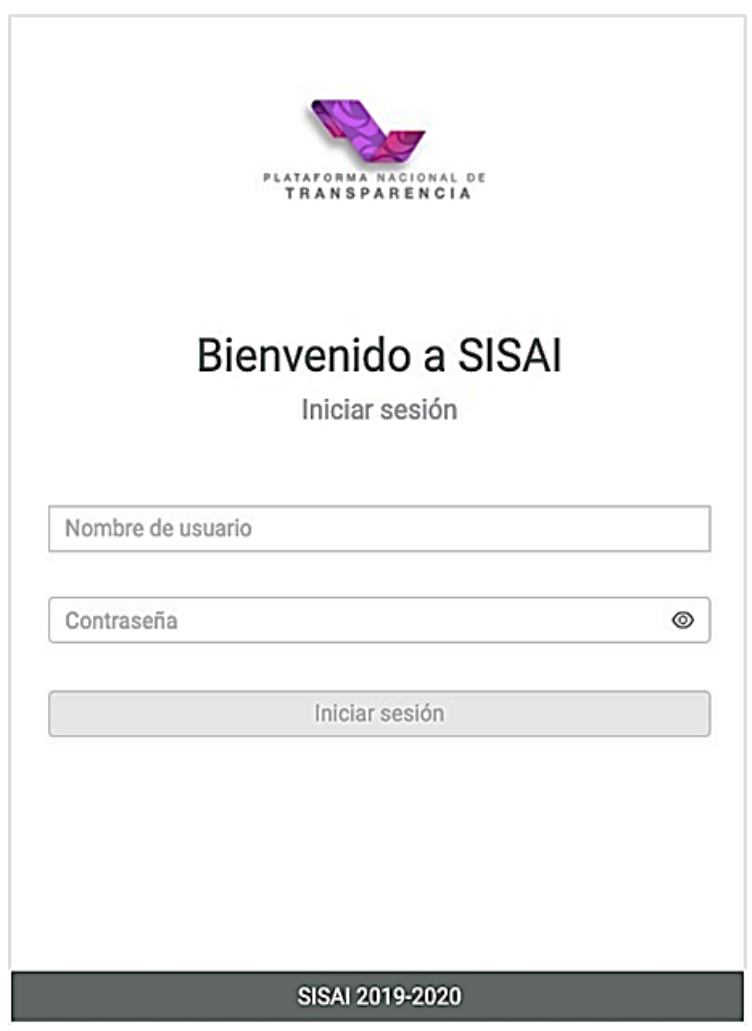

Figura 5 Pantalla de Acceso al sistema web Fuente: Elaboración propia

\section{- Módulo de Usuarios}

Permite el registro de los nuevos usuarios, la figura 6 muestra el formulario para agregar los datos, una vez requisitado, se da clic en el botón Registrar Usuario, los campos nombre, contraseña $y$ tipo son campos requeridos

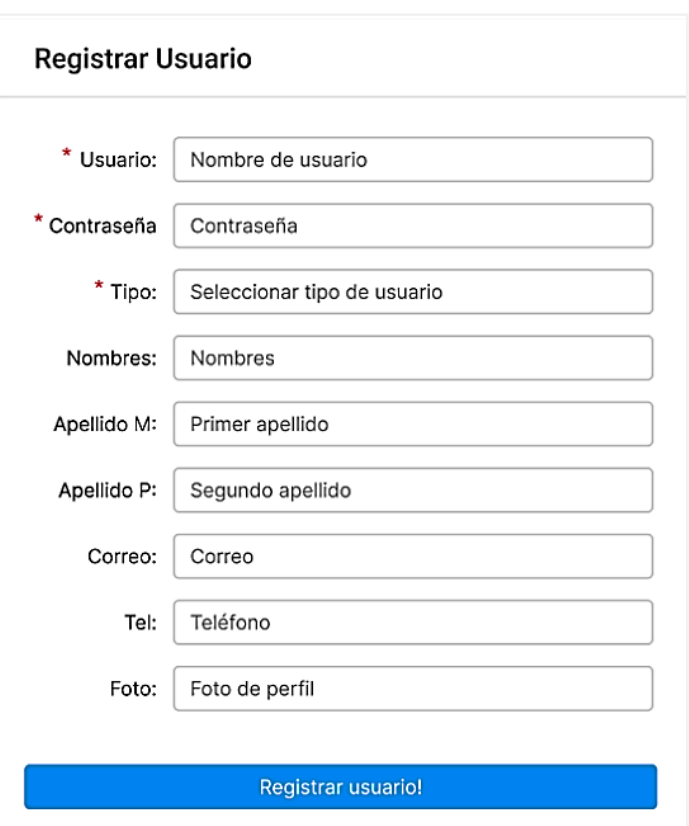

Figura 6 Módulo de registros de usuarios Fuente: Elaboración propia

\section{- Catálogo de Usuarios}

La interfaz muestra los usuarios ya registrados, como se aprecia en la figura 7.

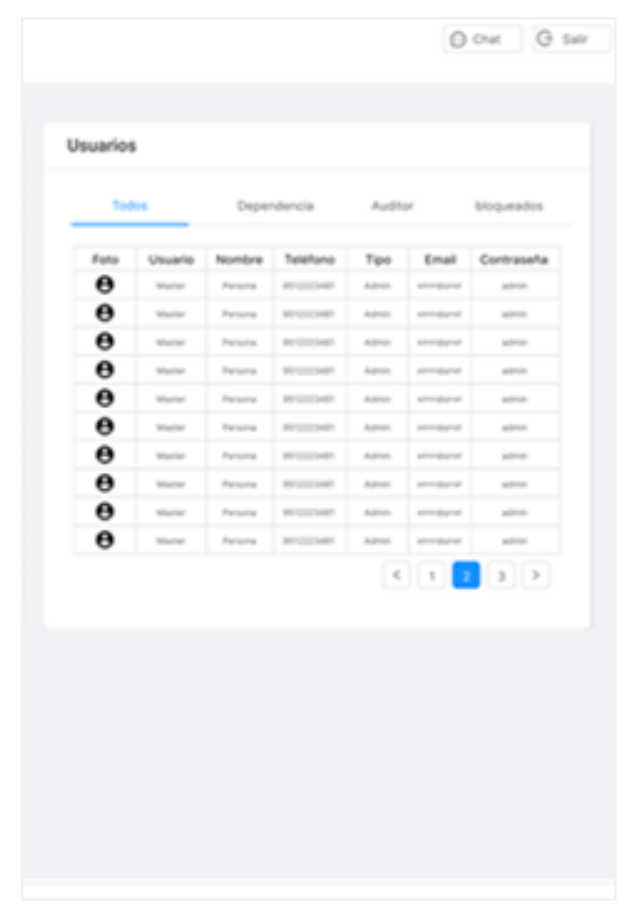

Figura 7 Catálogo de usuarios

Fuente: Elaboración propia

\section{- Módulo de Dependencias}

En este módulo se registran los datos de cada una de las dependencias del estado, los campos nombre y usuario son datos requeridos, una vez requisitado el formulario se da clic en el botón Registrar dependencia, como se visualiza en la figura 8. 


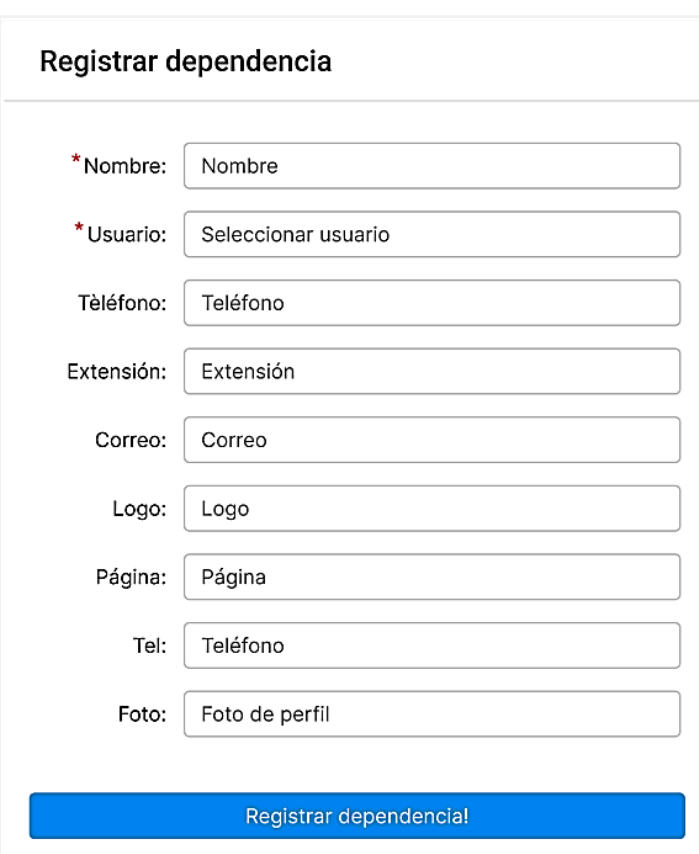

Figura 8 Módulo de registro de Dependencias Fuente: Elaboración propia

\section{- $\quad$ Módulo de Solicitudes}

Es el módulo principal del sistema web, donde el proceso de las solicitudes comienza al momento en que registra la solicitud por parte del responsable de la dependencia o de la propia Secretaría, en la figura 9 se muestra el estado, el número de folio, el solicitante, la fecha de inicio, la fecha de entrega y el nombre de la dependencia a quien se le está solicitando la información, además de las opciones.

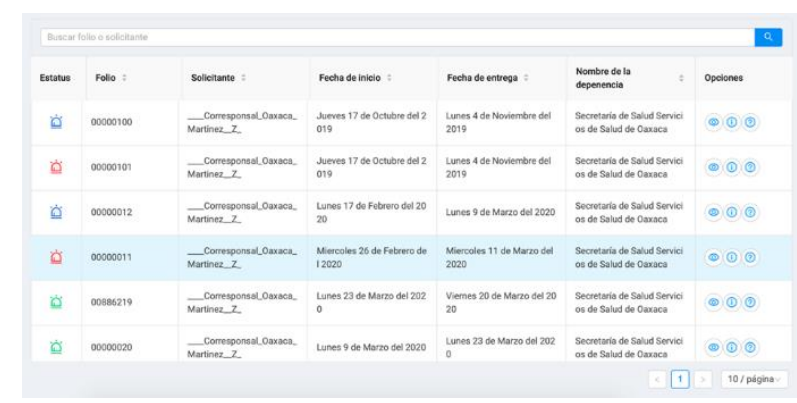

Figura 9 Módulo de Solicitudes

Fuente: Elaboración propia

\section{- $\quad$ Módulo de Seguimiento de Solicitudes}

Contiene la pantalla principal de las solicitudes de información, donde se muestra el estado del semáforo, los tipos de movimientos, los detalles y la línea el tiempo de la solicitud, como se visualiza en la figura 10 .

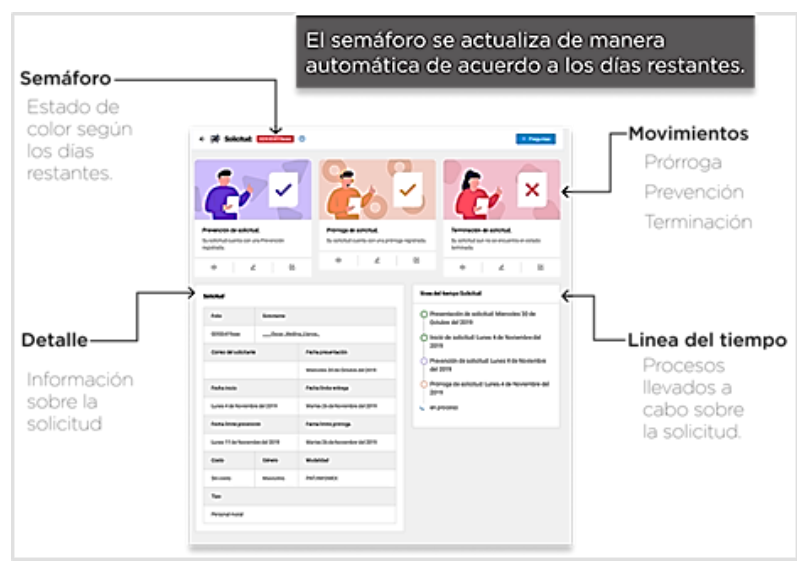

Figura 10 Pantalla principal del módulo de Solicitudes Fuente: Elaboración propia

En la figura 11, se presenta el seguimiento de la solicitud cuando se encuentra en prevención, en prórroga o cuando se da por terminada la solicitud, además permite registrar las preguntas.

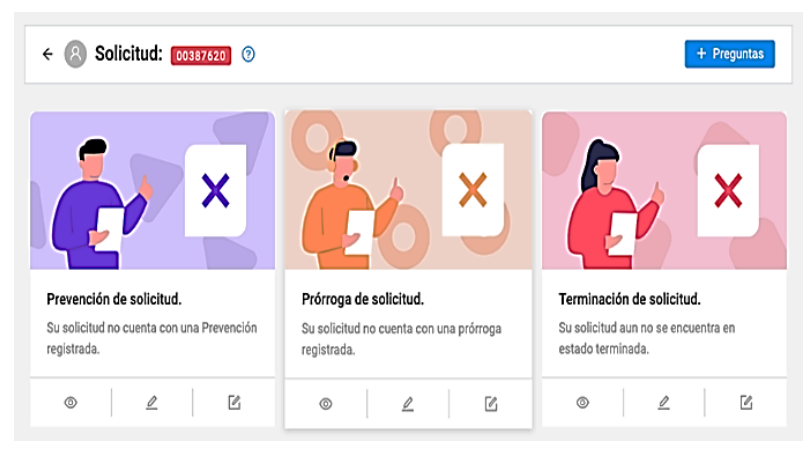

Figura 11 Módulo de seguimiento de Solicitudes Fuente: Elaboración propia

\section{- Módulo de Clasificador}

Este módulo permite clasificar las solicitudes con base a un clasificador principal mediante palabras claves, también se pueden agregar subclasificadores según sea necesario con el fin de que se tenga una organización adecuada de la información contenida en las solicitudes, como se muestra en la figura 12.

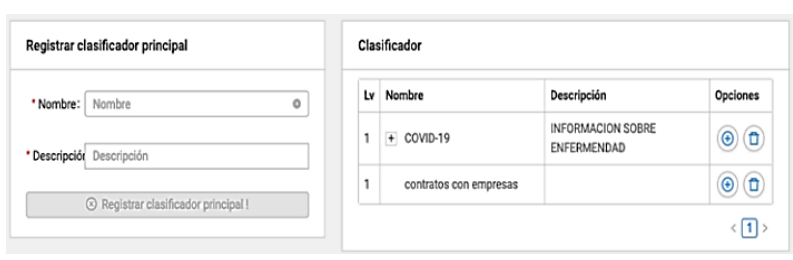

Figura 12 Módulo de clasificador Fuente: Elaboración propia 


\section{- $\quad$ Módulo de reportes}

En la figura 13, se muestra la pantalla con el informe anual de recepción de solicitudes de acceso a la información, y el botón para descargar el archivo en Excel.

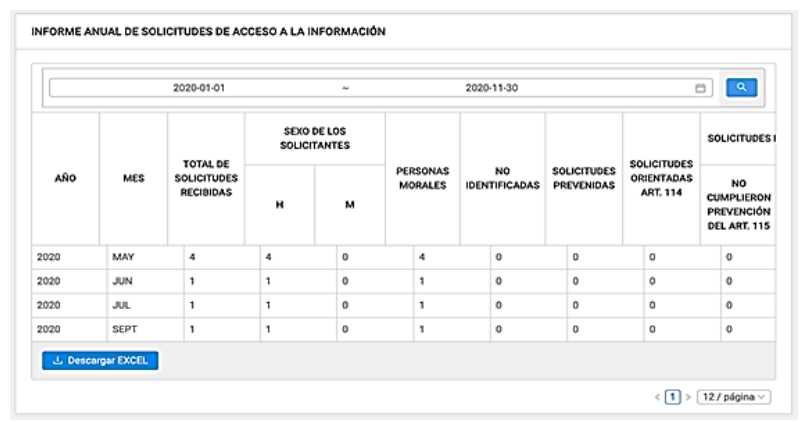

Figura 13 Generación del Informe anual

Fuente: Elaboración propia

También muestra las gráficas de las solicitudes recibidas por dependencia como se ve en la figura 14.

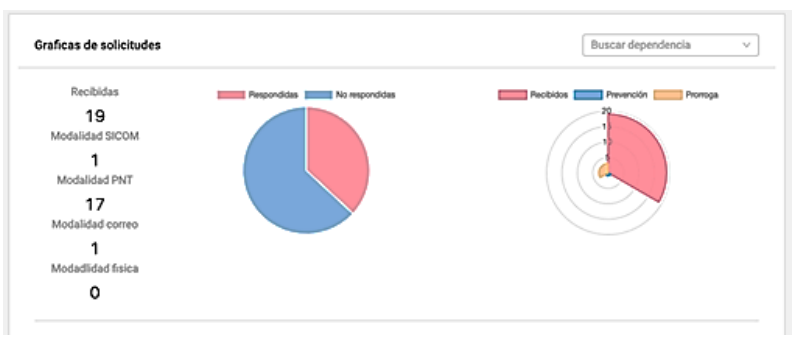

Figura 14 Graficas de Solicitudes

Fuente: Elaboración propia

\section{Agradecimientos}

A la Secretaría de Contraloría y Transparencia Gubernamental del Estado de Oaxaca.

Nuestro Reconocimiento a las siguientes personalidades:

Mtro. José Ángel Díaz Navarro

Secretario de la Contraloría y Transparencia Gubernamental

Lic. Máximino Vargas Betanzos

Subsecretario de Contraloría Social y Transparencia

Lic. José Manuel Méndez Spíndola

Director de Transparencia

Lic. Omar Pablo Mendoza
Enlace Informático de la Dirección de Transparencia

Por las facilidades, asesoría e interés que mostraron para la realización del presente trabajo, el cual se efectúo bajo un marco de colaboración entre el Tecnológico Nacional de México, Campus Oaxaca y la Secretaría de la Contraloría y Transparencia Gubernamental del Estado de Oaxaca.

Así mismo un agradecimiento al Tecnológico Nacional de México, que, a través del Campus Oaxaca, ha otorgado los espacios y recursos para el desarrollo del presente proyecto. Finalmente, se agradece también la colaboración y dedicación de los autores del artículo, profesores y estudiantes que han participado en la investigación hasta lograr los resultados que se presentan; cuyo objetivo es divulgar los hallazgos a la comunidad académica y al público en general sobre los trabajos que se desarrollan en nuestra Institución desde el punto de vista académico y educativo.

\section{Conclusiones}

Con el trascurrir de los tiempos, los sistemas de información han evolucionado la forma en cómo operan las instituciones, generando así ventajas competitivas, la implantación y desarrollo de los sistemas de información no resulta sencillo, requiere de una comunicación efectiva entre el cliente y el equipo de desarrollo para identificar adecuadamente los requerimientos y se cumplan con los tiempos de entrega en tiempo y forma.

Por otro lado, el desarrollo de los sistemas de información y las tecnologías de la información ha permitido eficientar las tareas en las organizaciones e instituciones, comparten los recursos, reducen los tiempos en el desarrollo de sus actividades, generando así el aumento de la productividad en las instituciones y dependencias. 
Con el Sistema Web de Solicitudes de Acceso a la Información la Secretaría de la Contraloría y Transparencia Gubernamental y las dependencias del estado, se benefician en gran medida; ya que cuenta con una base de datos que permite la organización eficiente de los datos y la información de todos los procesos, y módulos que conforman el sistema, el control y el seguimiento de las solicitudes, ya que apoyados de un semáforo de colores que se genera de manera automática permite saber en qué parte de la respuesta se encuentra la solicitud de acuerdo con los plazos legales establecidos por la ley, así mismo permite el registro de las dependencias, y la generación de los reportes tanto generales como anuales según sea necesario, el chat interno de comunicación entre las dependencias y la creación de las reuniones en caso de que sean necesarias para la toma de decisiones.

Cada dependencia es notificada en forma oportuna de algún evento, reunión o actividad pendiente por realizar y la atención brindada a los ciudadanos que realizan las solicitudes mejora; una de las ventajas competitivas que tiene el sistema web es que la información está disponible en tiempo y forma; información procesada y almacenada que ayuda a la Secretaria de Secretaría de Contraloría y Transparencia Gubernamental en la toma de decisiones.

\section{Referencias}

Avison D., Pries-Heje J. (2005). Research in information system: A handbook for research supervisors and their students (G. P. Publishing Ed.): Elsevier Butterworht- Heinemann.

Cobo, Á., Gómez, P., Pérez, D., \& Rocha, R. (2005). PHP y MySql: Tecnología para el desarrollo de aplicaciones web. España: Díaz de Santos.

Cohn, M. (2004). User Stories Applied for Agile Software Development. Boston: Pearson Education, Inc.

Collado, J., A., (s.f.). La Universidad Virtual. Recuperado el 24 de Septiembre de 2014, de Concepto de Sistema de Información en la organización:

http://dpacomputacionunpa.files.wordpress.com /2011/10/1_conceptos_de_si_en_organ izaciones.pdf.
Contreras, V., P., \& Ortiz, L. (2020). Abriendo los Partidos Políticos: acceso a la información tras la Reforma a la Ley Orgánica Constitucional de Partidos Políticos. Revista de derecho (Valdivia), 33(1), 253-272.

De Miguel, C., A., Piattini, V., M., G., y Marcos, M. E. (1999). Diseño de bases de datos relacionales. ISB N 84-7897-385-0. Madrid: RA-MA

Donovan, A., A., A., Kernighan, W., B., (2016). The Go Programming Language Copyright. Editorial, Addison-Wesley https://www.gopl.io/ch1.pdf

Fernández, A., (1998). Producción y diseño gráfico para la World Wide Web. Barcelona, España. Gallego, M. T. (s.f.).

Gary, W., Hansen., J., V., (2014). Diseño y Administracion de Bases de Datos. Mexico: Prentice Hall.

Laudon, K., \& Laudon, J., (2004). Sistemas de información gerencial (Octava ed.). México: Pearson

Luján, M., S., (2002). Programación de aplicaciones web: historia, principios básicos y clientes web. Editorial Club Universitario

Maddison, R. N. 1983. Information System Methodologies. Wiley Henden, 1983

Nevado, M. (2010). Introducción a Las Bases de Datos Relacionales. España: Visión Libros

Silberschatz, A., Korth, H.F. y Sudarshan, L.S.(2006). Fundamentos de bases de datos. $5^{\text {a }}$ edición. España: McGrawHill/INTERAMERICANA DE ESPAÑA, S.A.U.

Whitten, J, Bentley. L.D y K.C. Dittman, 2004. System análisis \& design methods citado por Fernández Vicenç, 2006: Desarrollo de sistemas de información: una metodología basada en el modelado. 


\section{Enlaces}

http://www.diputados.gob.mx/LeyesBiblio/pdf/

LGTAIP_130820.pdf

https://www.plataformadetransparencia.org.mx/ web/guest/glosariopnt

https://www.esic.edu/rethink/tecnologia/model o-entidad-relacion-descripcion-aplicaciones. 